SCIENTIFIC REPORT

\title{
Optical coherence tomography characterisation of idiopathic central serous chorioretinopathy
}

\author{
J A Montero, J M Ruiz-Moreno
}

Br J Ophthalmol 2005;89:562-564. doi: 10.1136/bjo.2004.049403

Aim: To describe retinal findings in patients with idiopathic central serous chorioretinopathy (ICSC) as assessed by optical coherence tomography (OCT), and to compare them to fluorescein angiography (FA) findings.

Methods: Case series of 39 eyes from 36 patients with ICSC. Complete ophthalmological examination, last generation OCT (StratusOCT, Software version 3.1) and FA were performed. Six radial scans using OCT were performed and repeated. Singular findings were recorded, OCT images were measured and the results compared with those of FA. The main outcome measures were FA and OCT findings. Results: Two patterns of distinct OCT findings are described. In the first one, an optically empty vaulted area of different heights was observed under the neurosensory retina in 36 eyes, being related to fluorescein filled areas; in 35 of them, highly characteristic small bulges could be observed protruding from the retinal pigment epithelium (RPE), angiographically related to leaking spots. In the second pattern, three eyes showed an almost semicircular space under the RPE, with thinner overlying retina.

Conclusions: OCT may offer a new approach to the staging and knowledge of ICSC, and may help the understanding of the mechanisms of the disease.

diopathic central serous chorioretinopathy (ICSC) typically affects young and middle aged males in their third to fifth decades. Patients may develop one or more small areas of serous detachment of retinal pigment epithelium (RPE) in the macula or in the paramacular area, which may be followed by serous detachment of the overlying and surrounding retina. Demonstration of ICSC is based on angiographic pooling of subretinal fluid, appearance of defects in the RPE, and typical dye leakage from the choroid into the subretinal space. ${ }^{12}$

New diagnostic and therapeutic tools have increased our ability to study this condition, allowing us to have new theories on its in-depth mechanisms or the presence of choroidal neovascularisation $(\mathrm{CNV}) .^{3}{ }^{4}$ Optical coherence tomography (OCT) is an imaging procedure that uses reflection of light off the retinal layers to create a false colour tomographic image of retinal and RPE structures with a resolution of $10 \mu \mathrm{m}$ axially and $20 \mu \mathrm{m}$ in transverse dimension. ${ }^{5}$

\section{METHODS}

We performed OCT (StratusOCT Software 3.1, Carl Zeiss Meditec Inc, San Leandro, CA, USA) and fluorescein angiography (FA) (Imagenet 2000, Topcon TRC50IX, Topcon Corp, Japan) on 39 eyes from 36 consecutive patients with ICSC. OCT and FA were performed on the same day.
Eleven patients ( 10 males, one female, 13 eyes) had had symptomatic ICSC with angiographic changes for more than 12 months without clinical normalisation (chronic forms). Fifteen patients ( 11 males and four females, 15 eyes) have had the first episode for less than 4 months (acute forms). Ten patients (six males and four females, 11 eyes) have had two or more episodes.

OCT was performed by acquiring six radial scans, $6 \mathrm{~mm}$ long, centred in the fovea using the fast macular scan function (transverse sampling, 128 A-scans). When fixation was poor, scans were centred in the fovea under video surveillance. OCT scans were repeated three times to ensure reproducibility. When particular details were observed, the scan was centred in that location to check that they were not caused by saccadic movements or other artefacts, at a higher scan rate (transverse sampling, $512 \mathrm{~A}$-scans).

Scans were examined and compared to FA to determine the mutual relation and nature of the findings. OCT video imaging was used to centre scans on angiographic photographs to locate and identify the findings on FA. Retinal thickness was measured at the fovea using the macular thickness calliper.

\section{RESULTS}

The mean age of the patients was 43 (SD 8) years, (range 2968 ), with 27 males (75\%) and nine females (25\%). All were white. Mean visual acuity at baseline was 20/25 (range 20/20 to $20 / 60$ ). Fundus examination showed serous detachment in all of them with patches of RPE atrophy. No haemorrhages or choroidal neovascularisation (CNV) were observed.

OCT showed foveal distortion and cystic and atrophic macular changes in all eyes. Thirty six eyes (92\%) showed neuroretinal detachments corresponding to the affected area, with empty spaces underneath. Three eyes (8\%) showed RPE detachment and overlying retinal thinning.

Two different patterns could be observed. The first pattern was an empty vaulted area of variable height (41-539 $\mu \mathrm{m}$, mean $198 \mu \mathrm{m}$, SD 107) under the neurosensory retina, angiographically related to fluorescein filled areas. It was present on 36 eyes ( 15 from acute forms $100 \%$, 11 from multiepisodic forms $100 \%$, and 10 from chronic forms $77 \%$ ). Empty spaces were underlined by a hyper-reflective line, probably corresponding to scarred RPE. In 35 eyes a small bulge was observed protruding from RPE (fig lA-D) with hyper-reflective cortex and less hyper-reflective core measuring 16-70 $\mu \mathrm{m}$ wide, rising $10 \mu \mathrm{m}$ from the RPE. In 10 eyes there were two bulges, but in 25 cases there was only one. Angiographically these bulges were related to leaking spots (fig 2A-D). Foveal thickness was 135-268 $\mu \mathrm{m}$ (mean $207 \mu \mathrm{m}, \mathrm{SD} 34)$. In four cases the cystic space was very

Abbreviations: CNV, choroidal neovascularisation; FA, fluorescein angiography; ICSC, idiopathic central serous chorioretinopathy; OCT, optical coherence tomography; PDT, photodynamic therapy; RPE, retinal pigment epithelium 


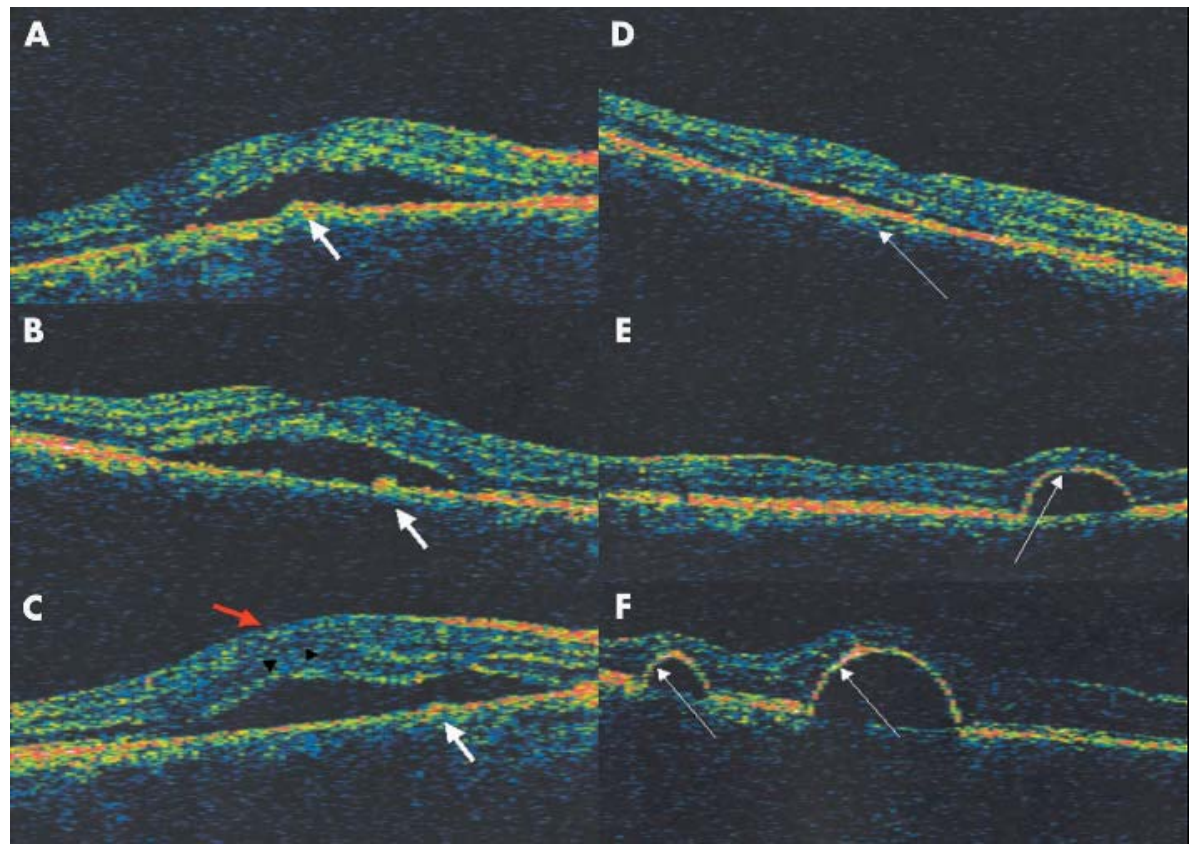

Figure 1 (A-C) Optical coherence tomography (OCT) in acute forms shows bulges protruding from retinal pigment epithelium (RPE) layer under an optically empty space. Integrity of RPE underneath shows absence of RPE detachment. (C) Shows foveal distortion (lack of foveal pit, red arrow) and intraretinal cysts (arrowhead). (D) OCT shows shallow fluid accumulation with hyper-reflective underlying RPE (arrow). (E) and (F) OCT in chronic forms shows vaulted sub-RPE spaces, proving the presence of RPE detachment (arrows). Overlying retina is thinner than normal. Note RPE difference compared to (A)(C) in which RPE remains in place. shallow (fig 1D), showing the previously described bulge. Only one case showed one small cystic structure with an empty core, which might be assimilated to a small cystic RPE detachment.

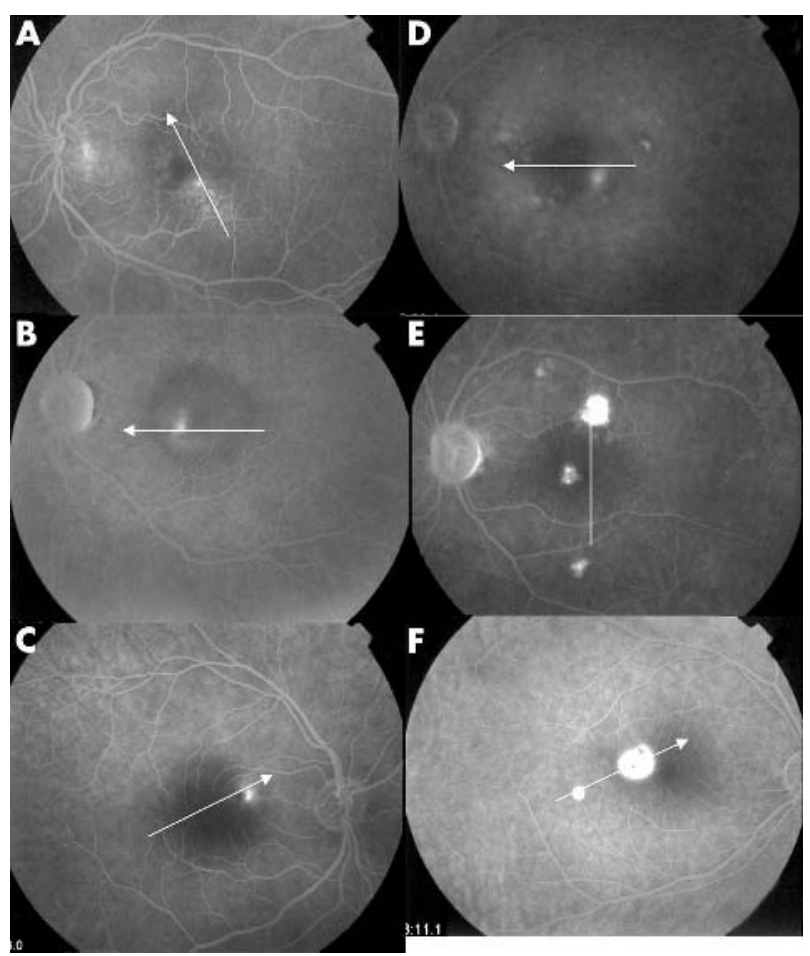

Figure $2(A-C)$ Fluorescein angiograms (FA) of eyes shown in figure $1 \mathrm{~A}-\mathrm{C}$, respectively. Arrows show the direction of OCT scans. Note the correspondence of angiographic leaking spots with OCT bulges previously described. (D) FA of eye in figure 1D. Atrophic and hypertrophic patches in RPE can be seen in the macula. Arrow shows the direction of OCT scan. (E) and (F) Show FA of eyes in figure 1D and E, respectively. Note the accumulation of fluorescein corresponding to RPE detachments in OCT in figure 1. Arrows show the direction of OCT scans.
Three eyes (all from chronic forms, 23\%) showed a vaulted space of variable height (fig lE, F) under RPE, what probably identifies them as RPE detachments. Overlying retina measured 83-145 $\mu \mathrm{m}$ (mean $106 \mu \mathrm{m}$, SD 36). Angiographic correspondence of these structures was actually RPE detachments (fig 2D and E).

FA showed fluorescein pooling under detached retina in all cases. Thirty six eyes (92\%) showed active forms of ICSC. Leaking points and smokestacks could be demonstrated in 28 eyes $(72 \%$ ) (fig $2 \mathrm{~A}-\mathrm{D})$; meanwhile eight eyes $(20 \%)$ showed diffuse patterns with severe, diffuse RPE atrophy (fig 2E and F). One eye (3\%) showed only RPE defects. All eyes showed parafoveal, patchy RPE atrophy without CNV.

Comparing OCT scans with FA it could be demonstrated that protrusions were located exactly where fluorescein leakage appeared. A similar topographic relation could be established for RPE detachments.

\section{DISCUSSION}

The role of OCT as a non-invasive way of studying the retina is well known. ${ }^{35}$ 6-10 Several papers have recently appeared describing OCT findings in eyes with ICSC. ${ }^{3-14}$

We describe two different OCT patterns in patients with ICSC, and one finding highly characteristic of this condition. Acute and multiepisodic forms of ICSC showed in all cases empty subretinal spaces. In most of them bulges protruded from the RPE under a detached retina; this also appears in some chronic cases, which probably meant activity of the disease. Their location was that of the angiographic leakage.

The appearance of high, vaulted subretinal spaces in OCT was associated with the biomicroscopical presence of local neuroretinal detachment and fluorescein filled areas, Bulges could not be located biomicroscopically.

Twenty three per cent of chronic forms appeared to be associated with high RPE detachments and retinal thinning without retinal detachment. FA showed progressive filling. Biomicroscopically they showed well defined neuroretinal detachment.

Kamppeter and Jonas ${ }^{8}$ and Drexler et al have previously described similar findings lacking hyper-reflective RPE, considering them to be RPE detachments. Drexler et al were uncertain whether these findings might actually be 
artefactual. We cannot consider these bulges as RPE detachments since RPE seemed to be still adhered under them, nor artefactual because they are reproducible and correlate with FA leaking points. We would rather consider them RPE protrusions, or thickening.

Different findings have been described in eyes with ICSC. $^{12}{ }^{14}$ Wang et $a l^{12}$ described retinal thinning ranging from $51-74 \%$ of normal fovea in patients with longer lasting ICSC. Iida et $a l^{14}$ described retinal thickening as retinal oedema similar to what is found in patients with CNV associated with AMD. ${ }^{15}{ }^{16}$ In our series, retinal thickness observed during the acute phase is not much different from that of healthy volunteers $(219.8 \mu \mathrm{m})$, and was clearly inferior to what is found in patients with active CNV (289.9 (SD 92.1) $\mu \mathrm{m}) .{ }^{15}$ It must be also borne in mind that different software and hardware were used in Iida's work, ${ }^{14}$ which may also contribute to the different absolute measurements.

ICSC is considered a multifactorial disease in which focal defects of RPE origin leak into the subretinal space, and there are secondary RPE and retinal changes. ${ }^{17}$ The mechanism of ICSC is highly controversial. ${ }^{18}$ RPE serous detachment appears at early phases, and usually can be angiographically identified as a leaking point. ${ }^{1}$ RPE detachment is usually associated to serous detachment of the overlying retina. Some attempts have been made to treat ICSC by photodynamic therapy (PDT). ${ }^{411}$

Pathological material is limited. Serous macular detachment has been pathologically described, with and without defects or detachment of the RPE. ${ }^{1}$

Ultrastructural studies in animal models of ICSC have shown defects in RPE, which might favour a breakdown in the outer blood-retinal barrier. ${ }^{19}$

The issue is whether the bulges we describe are local RPE abnormalities related to FA leakage and to the pathophysiology of ICSC, or artefacts. We consider that the close topographic correspondence of these structures in FA and OCT suggest they are probably involved in the mechanism of the condition. We have found them in 35 of 39 eyes with ICSC $(90 \%)$, but not in more than 1800 patients (more than 2600 eyes) with conditions different from ICSC (normal, CNV, uveitis, macular oedema, optic disc pit, etc). They might represent minimal RPE detachments, or focal defects of RPE.

Chronic forms were associated with retinal thinning and highly vaulted RPE detachments like those described by Kamppeter and Jonas ${ }^{8}$ and Drexler et al. ${ }^{9}$ The persistence of subretinal fluid may induce retinal cystoid changes and thinning, mainly in chronic or recurrent forms. ${ }^{1}$

Considering ISCS angiographic patterns (hot spot $72 \%$, diffuse RPE atrophy in up to $90 \%$ of cases), OCT seems to be more sensitive to give a diagnosis (bulge 90\%, focal neuroretinal or RPE detachment up to $100 \%$ ), and to evaluate the degree of activity of ICSC, and is not influenced by the degree of RPE atrophy. It also provides an easier differential diagnosis from other conditions which might be similar by biomicroscope and FA (such as age related macular degeneration). OCT is safer and less time consuming than FA, and can be repeated more often.
OCT may offer a new approach to the diagnosis and evaluation of retinal damage in ICSC, and help us to understand its staging and mechanisms.

\section{Authors' affiliations}

J A Montero, J M Ruiz-Moreno, Instituto Oftalmológico de Alicante, Vitreo-Retinal Unit, Alicante, Spain

J M Ruiz-Moreno, Department of Ophthalmology, Miguel Hernández University School of Medicine, Alicante, Spain

The authors have no financial interest in the devices and procedures described.

Correspondence to: Javier A Montero, Instituto Oftalmológico de Alicante, Avenida de Denia 111, 03015, Alicante, Spain; msm02va@ wanadoo.es

Accepted for publication 1 October 2004

\section{REFERENCES}

1 Gass JDM. Specific diseases causing disciform macular detachment. In: Stereoscopic atlas of macular diseases: diagnosis and treatment. St Louis, Mosby, 1997:49-70.

2 Kitaya N, Nagaoka T, Hikichi T, et al. Features of abnormal choroidal circulation in central serous chorioretinopathy. $\mathrm{Br} J$ Ophthalmol 2003;87:709-12.

3 He SZ, Wang W, Li XL, et al. Optical coherence tomography features of central exudative chorioretinopathy. Zhonghua Yan Ke Za Zhi 2003:39:669-72.

4 Cardillo Piccolino F, Eandi CM, Ventre L, et al. Photodynamic therapy for chronic central serous chorioretinopathy. Retina 2003;23:752-63.

5 Hee MR, Puliafito CA, Duker JS, et al. Topography of diabetic macular edema with optical coherence tomography. Ophthalmology 1998;105:360-70.

6 Goebel W, Kretzchmar-Gross T. Retinal thickness in diabetic retinopathy. A study using optical coherence tomography (OCT). Retina 2002;22:759-67.

7 Rogers AH, Martidis A, Greenberg PB, et al. Optical coherence tomography findings following photodynamic therapy of choroidal neovascularization. Am J Ophthalmol 2002;134:566-76.

8 Kamppeter B, Jonas JB. Central serous chorioretinopathy imaged by optical coherence tomography. Arch Ophthalmol 2003;121:742-3.

9 Drexler W, Sattmann H, Hermann B, et al. Enhanced visualization of macular pathology with the use of ultrahigh-resolution optical coherence tomography. Arch Ophthalmol 2003;121:695-706.

10 lida T, Yannuzzi LA, Spaide RF, et al. Cystoid macular degeneration in chronic central serous chorioretinopathy. Retina 2003;23:1-7.

11 Taban M, Boyer DS, Thomas EL, et al. Chronic central serous chorioretinopathy: photodynamic therapy. Am J Ophthalmol 2004; 137:1073-80.

12 Wang MS, Sander B, Larsen M. Retinal atrophy in idiopathic central serous chorioretinopathy. Am J Ophthalmol 2002;133:787-93.

13 Otsuka S, Ohba N, Nakao K. A long-term follow-up study of severe variant of central serous chorioretinopathy. Retina 2002;22:25-32.

14 lida T, Hagimura N, Sato T, et al. Evaluation of central serous chorioretinopathy with optical coherence tomography. Am J Ophthalmol 2000;129:16-20

15 Montero J, Ruiz-Moreno JM. Macular thickness in patients with choroidal neovascularization determined by RTA and OCT 3. Comparative results. Eye $2005 ; 19: 72-6$

16 Montero J, Ruiz-Moreno JM, Tavolato M. Follow-up of age related macular degeneration patients treated by photodynamic therapy with optical coherence tomograph 3. Graef Arch Clin Ophthalmol 2003;241:797-802.

17 Spitznas M. Central serous retinopathy. In: Ryan SJ, Schachat AP, Murphy RP, Paty A, eds. In: Retina. St Louis: CV Mosby, 1989;2:217-27.

18 Guyer DR, Gragoudas ES. Central serous chorioretinopathy. In: Albert DM, Jakobiec FA, eds. In: Principles and practice of ophthalmology. Philadelphia: Saunders Company, 1994:818-25.

19 Yoshioka H, Katsume Y, Akune H. Experimental central serous chorioretinopathy in monkey eyes: fluorescein angiographic findings. Ophthalmologica 1982;185:168-78. 